\title{
Condiciones de demanda turística en una ruta ecoturística en el corredor Coveñas-Tolú en el departamento de Sucre $^{1}$
}

Conditions of tourist demand in an eco tourist route in the corridor Coveñas-Tolú in the department of Sucre

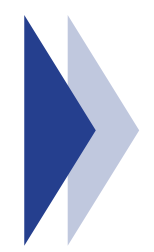

Erika Patricia Ruiz Carta

Administradora de Empresas, Joven Investigador Programa de Formación de Alto Nivel Departamento de Sucre, Corporación Universitaria del Caribe, Sucre-Colombia

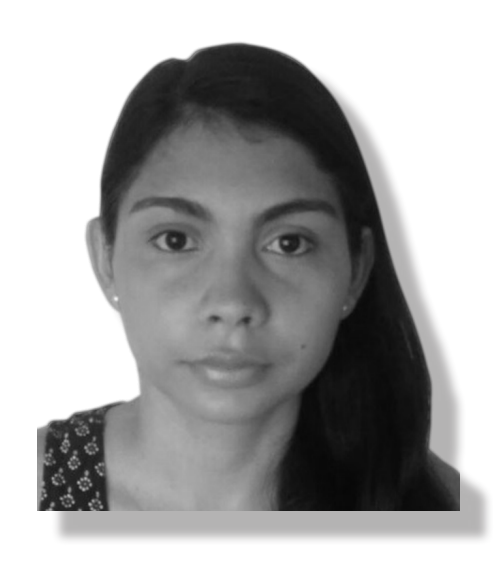

1. Este artículo es resultado de la investigación denominada "Diseño de una Ruta Ecoturística en el corredor:

Coveñas-Tolú, Departamento de Sucre 2016". Investigación llevada a cabo en el marco de la convocatoria ejecutada por la Gobernación de Sucre Joven Investigador Mariscal Sucre 2015 en convenio con el Programa de Formación de Alto Nivel (FAN) de la Corporación Universitaria del Caribe, CECAR. 
Andrés Vergara Narváez

Ingeniero agroindustrial, especialista en gerencia de proyectos, especialista en logística, Magister en Dirección de Operaciones y Logísticas, Corporación Universitaria del Caribe- CECAR

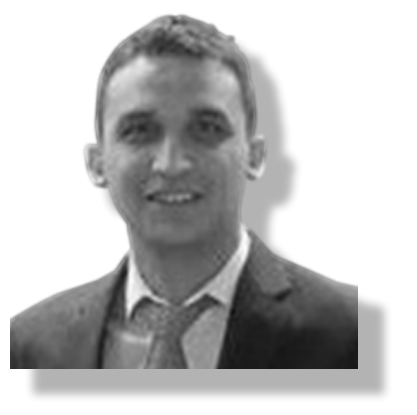

RESUMEN

El presente artículo tiene como finalidad describir las condiciones de demanda turística en la creación de rutas ecoturísticas en los municipios Coveñas y Tolú. El tipo de estudio es descriptivo. En el proceso investigativo se tomó una muestra estratificada de 232 turistas y 37 establecimientos de alojamiento; además se utilizó un grupo de profesionales para valorar áreas naturales según la metodología del Mincit (2010) y la aplicación de encuestas directas a los turistas para recopilar la información. A partir del estudio se determinó que la demanda de los territorios es de carácter nacional, con el 54\% de turistas provenientes del interior del país, y, a su vez, se encontró que el 70\% de los turistas que visitan los destinos realizan actividades vinculadas a sol y playa. Sin embargo, cerca del 30\% están dispuestos a practicar el turismo de naturaleza. Así mismo que sólo se consideraran 2 áreas para practicar el "ecoturismo", por las condiciones de conservación y accesibilidad a las mismas. Se concluye que se hace necesaria la implementación de estrategias encaminadas a mejorar las condiciones de demanda, aprovechando el potencial del recurso natural y diversificando la oferta de servicios que contribuyan a mejorar el nivel de los destinos.
Abstract

This article aims to describe the conditions of tourism demand in creating ecotourism routes in municipalities: Covenas and Tolu. The type of study is descriptive. In the research process a stratified sample of 232 tourists and 37 accommodation establishments was taken; plus a group of professionals was used to assess natural areas according to the methodology of Mincit 2010 and the application of direct tourists surveys to gather information. From the study it was determined that the demand for the territories is national with 54\% coming from inside the country, turn that $70 \%$ of tourist destinations are active tourism: sun and beach. However about 30\% are willing to practice nature tourism. Also only 2 areas were considered to practice "ecotourism" by the conditions of preservation and accessibility to them. It is concluded that the implementation of strategies aimed at improving demand conditions is necessary, taking advantage of the potential of the natural resources and diversifying the range of services that help improve the level of destinations.

Keywords: Tourist routes, tourism demand, tourism services, natural areas.

Palabras Clave: Rutas ecoturísticas, demanda turística, servicios turísticos, áreas naturales. 


\section{Introducción}

El sector turismo ha adquirido una importancia significativa en la economía del país, como polo de desarrollo en los ámbitos social, económico, cultural y ambiental. Actualmente el turismo de naturaleza, se convierte en una apuesta productiva, contemporánea, que agrega valor y permite la preservación de zonas naturales y mantiene la identidad cultural de una comunidad.

La subregión Golfo de Morrosquillo se constituye como la principal área turística del departamento de Sucre, e involucra los municipios de Coveñas y Tolú localizados a $57 \mathrm{~km}$ y a $50 \mathrm{~km}$ de Sincelejo respectivamente, por presentar características naturales para desarrollar actividades del ecoturismo y por ser áreas de mayor influencia de visitas por los turistas. La investigación se centró en conocer las condiciones de demanda turística en el diseño una ruta eco turística que permita promover dichos territorios como destinos turísticos en relación con la práctica del ecoturismo.

El presente artículo tiene como objetivo determinar las condiciones de demanda turística en una ruta ecoturística en el corredor Coveñas-Tolú, en el departamento de Sucre; donde se da inicio al proceso investigativo con la revisión de los antecedentes, en los cuales se recopilan los aportes de autores que orientaron el estudio de rutas. Seguido, se procede a la exploración de fuentes bibliográficas sobre turismo, ecoturismo, desarrollo endógeno, sostenible, planes de desarrollo, demanda turística, la normatividad de la actividad turística y las características generales de los municipios caso de estudio; por otra parte la metodología utilizada para recolectar la información de fuentes primarias, como la valoración de recursos naturales, mediante la metodología del MINCIT (2010), aplicación de instrumentos de caracterización dirigidos a turistas de la zonas con el fin de conocer las condiciones de demanda turística de los territorios. Finalmente, se presentan las conclusiones que permiten cumplir con el objetivo de caracterizar y conocer los criterios de demanda en la creación de rutas ecoturísticas.

\section{Fundamentos teóricos}

Conforme a Serrano, "el sector servicios es el principal sector económico en las economías de los países desarrollados. Incluye actividades tan diversas como la educación, el comercio, el turismo o los servicios personales [...] El sector servicios es el que más volumen de empleos genera y el que más transacciones comerciales registra" (2011, p, 1).

El sector Turismo se ubica en el sector terciario "servicios" de la economía y según la OMT lo define como: "un fenómeno social, cultural y económico relacionado con el movimiento de las personas a lugares que se encuentran fuera de su lugar de residencia habitual por motivos personales o de negocios/ profesionales" (Organizacion Mundial de Turismo, OMT, 2007). El campo del turismo presenta particularidades en las diferentes categorías que tiene, las cuales las determina las condiciones de los territorios, en este caso se habla de la subcategoría ecoturismo, que de acuerdo con la Sociedad Internacional de Ecoturismo define el término como: "el viaje responsable a las áreas naturales para conservar el medio ambiente y mejorar el bienestar de las personas locales" (The International Ecotourism Society, 1990).

Durante los últimos años la economía en el departamento de Sucre se ha centrado en el sector terciario especialmente en las ramas de actividad servicios y comercio, "el sector terciario dela economía ha crecido en promedio entre 2000 y 2011 a nivel departamental a una 
tasas del 4,6\% por encima del crecimiento de este sector a nivel nacional que fue de 4.3\%" (Comts, 2013). De acuerdo a esto, el departamento posee municipios con riquezas naturales, culturales para ser promocionados y potenciados paralelamente con otros tipos de turismo que ofrezcan productos certificados y de alta calidad con el objetivo de aumentar la afluencia de turistas a las zonas de Coveñas y Tolú.

Ahora bien, el sistema turístico está conformado por la demanda y oferta turística que tienen un papel importante en el desarrollo de una zona o destino de cualquier país. Según Rigol \& Pérez (2011, p,1), La demanda turística es "el conjunto de turistas actuales y potenciales que desean, pueden y están dispuestos a disfrutar de las facilidades, atractivos, actividades, bienes y (o) servicios turísticos en función del precio y su renta”. En los territorios la demanda está dada por una serie de condiciones que los turistas necesitan para pasar más tiempo y disfrutar de las actividades, comodidades que ofrecen, la cual va de la mano con los servicios prestados por la oferta turística y que en conjunto definen las experiencias de las personas que eligen viajar a los destinos. Dicha demanda se desarrolla y fomenta en conjunto con los actores locales aprovechando la riqueza natural y cultural para tener una posición competitiva para los territorios de Coveñas y Tolú.

Según Alburquerque

El desarrollo local es el proceso de transformación de la economía y de la sociedad local, encaminado a mejorar las condiciones de los habitantes, a través de la actuación concertada entre los actores públicos y privados que permitan el aprovechamiento eficiente y sustentable de todos los recursos con los que cuentan y generar la creación de capacidades empresariales locales y ambientes innovadores en dicho territorio. (1997, p. 30)
El desarrollo local depende de como articular el manejo adecuado de los recursos existentes y con mayor potencial hacia la satisfacción de las necesidades de la comunidad local.

Así mismo, Vanegas (2006) señala que el proceso de integración tiene como objetivo la valoración del area de las comunidades, y su participación en el mercado del ecoturismo, en la conservacion y en el desarrollo de su comunidad . Por lo cual se hace importante que en la sostenibilidad, el desarrollo de los productos ecoturisticos debe estar sostenido en el conocimiento de los recursos y espacios (naturales, culturales) de las zonas de visitas, para establecer los límites de uso y los códigos de comportamiento como punto de inicio para el diseño de la oferta comercial. (Vanegas, 2006)

Por otra parte, el ecoturismo se considera una nueva modalidad de destino que apunta a la relación sostenible entre humanonaturaleza, fortaleciendo procesos de desarrollo económico, social, de identidad cultural y religiosa de cualquier país. Por tanto, la planificación de los productos ecoturisticos debe estar integrados los principios de planeación y gestión de los elementos naturales, materiales para su desarrollo; lo que implica tener definido de acuerdo al Рвот los espacios definidos para el uso de dichas actividades turisticas, el acceso y la capacidad de carga de las zonas, la oferta y las condiciones en la prestación del servicio, el pago compensatorio por explotar esas áreas tanto turistas como para los operadores y la participación de las comunidades locales.

De igual manera, el departamento de Sucre, cuenta con la subregión Golfo de Morrosquillo en donde se identifican zonas para la práctica de actividades del turismo de naturaleza: ecoturismo; los municipios de Tolú y Coveñas cuenta con atractivos como: mar, ríos, ciénagas, bosques de manglares, 
cuevas, extensas sabanas, variada y rica fauna. Características geográficas con ventajas comparativas que se convierten en puntos principales de afluencia de turistas en las temporadas altas (noviembre-diciembreenero-abril-junio-octubre), por lo que tienen todo un potencial para ser incluidos dentro de un producto ecoturístico estructurado, diferencial y que los posicione dentro del territorio nacional para turistas nacionales e internacionales. Consecuentemente, la política turística de Colombia tiene como objetivo mejorar la competitividad de los destinos y los productos, e incrementar la participación de la comunidad en la prestación de los servicios turísticos, en donde se pretende que los habitantes perciban un crecimiento en sus ingresos para que contribuya a la generación de riqueza de la zona y que a su vez se logre una distribución más equitativa y un aumento en la calidad de vida de los nativos.

Paralelo a esto, los planes de desarrollo de los municipios de Tolu y Coveñas, han integrado la línea estratégica de turismo como elemento dinamizador de la economía y calidad de vida de sus habitantes, estableciendo programas para el turismo cultural- patrimonial, naturaleza, sol y playa desde lo local para aprovechar las ventajas comparativas de la oferta como: cultura local, festividades, gastronomía, artesanias, folclor, áreas naturales para promover lineas de acción encaminadas a fortalecer este ámbito. Así mismo contemplan la sostenibilidad turística haciendo hincapié en las zonas naturales, sector de playas y la situación actual en la cual se encuentra, para ello propone estrategias para mitigar el impacto ocasionado por la influencia de las personas y empresas de la zona.

La correcta articulación entre los planes de desarrollo establecidos por los entes municipales y el empalme con las comunidades es lo que va a permitir que se sienten las bases del desarrollo, entiendo que son éstas mismas las que necesitan y quieren seguir aportando a su identidad cultural, por que primero se requiere conocer la cultura y expresiones nativas de un pueblo para despues poder hablar de turismo y de sus recursos y/o atractivos.

\section{Metodología}

Diseño: la investigación se realizó a partir de un enfoque cuantitativo, el cual permite recolectar, analizar la información encontrada, así como también a través de la observación directa en la valoración de sitios naturales (Mincit, 2010). De igual manera es de tipo descriptivo, puesto que se enfoca en determinar las condiciones de la demanda turística en áreas de interés y de los turistas, para delimitar así el problema de la investigación. En ese orden de ideas, se utilizó tanto fuentes primarias como secundarias para conseguir la información, que ofrecen datos oficiales, confiables.

Población: la población estuvo compuesta por los turistas que visitan los territorios de Coveñas y Tolú. Se utilizó la teoría del muestreo aleatorio estratificado puesto que la población se halla repartida en dos municipios. Debido a que no hay información estadística y datos concretos de la cantidad de turistas que ingresan a los territorios, se estimó una población de 30.000 visitantes con base en información suministrada por las secretarias de turismo; por consiguiente para determinar la cantidad de encuestas a aplicar y la repartición de las mismas, se determinó por la variable tipo de turismo que prefieren practicar los turistas y se realizaron 232 encuestas a turistas.

Instrumentos: la investigación involucra en la metodología la aplicación de varios instrumentos, se empleó la observación directa para la valoración de sitios naturales 
de conformidad con la metodología establecida por el Ministerio De Comercio, Industria y Turismo (2010), a cargo de un grupo interdisciplinario de profesionales, quienes evalúan las condiciones de las áreas objeto de estudio. Del mismo modo se aplicó una encuesta directa dirigida a los turistas de la zona para conocer y determinar los criterios de demanda en relación con el desarrollo de productos y/o actividades del ecoturismo.

\section{Resultados}

Los principales resultados en el estudio evidencian las características de los turistas que visitan las áreas naturales delos territorios. En cuanto a la procedencia del turismo, se ha visto incrementado las visitas de turistas de casi todo el país. Según la encuesta el 54\% de las personas provienen de las ciudades de Medellín, Bogotá y Cali; mientras que el $47 \%$ proceden de la región Caribe, por lo que se determina que la demanda es de carácter nacional en los municipios de Coveñas y Tolú.

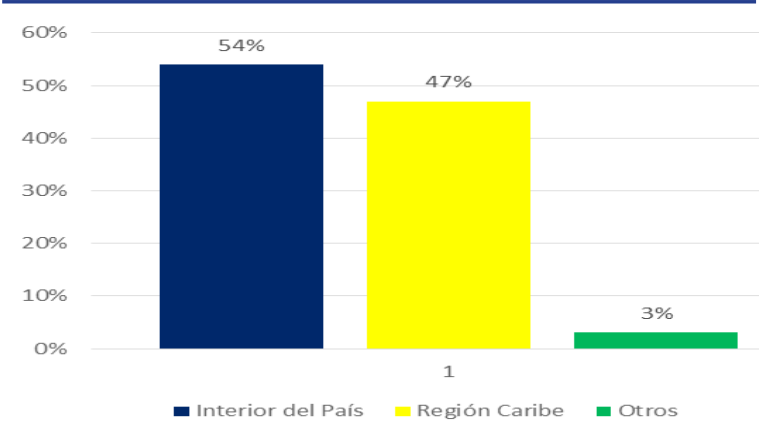

Figura 1. Procedencia de los turistas.

Fuente. Encuesta elaborada en el estudio Diseño de una Ruta Ecoturística en Tolú-Coveñas, 2016.

Esta clase de turistas, tienen como finalidad viajar a diferentes destinos para buscar actividades de sol, playa, naturaleza, descanso, diversión; tendencia que ha aumentado en los últimos años por lo que se pueden apreciar turistas de departamentos como Casanare y Santander, por ejemplo.
Por otra parte, en lo que se refiere a los motivos e intereses de los turistas para seleccionar los destinos de Coveñas y Tolú, en primer lugar, está el descanso, razón primordial para el $81,90 \%$ de los viajeros. En segundo lugar, se encuentra visitas familiares, motivación con el $17,67 \%$. La realización de negocios y trabajo representó el o,43\% de las personas que visitaron los destinos en el 2016.

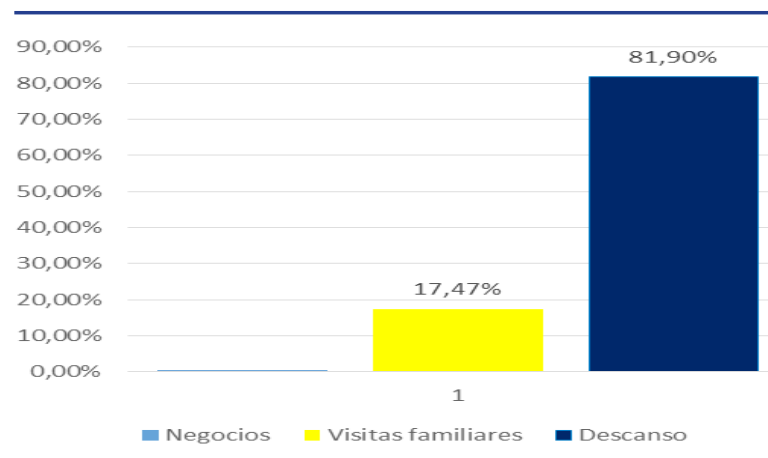

Figura 2. Motivo principal de viaje.

Fuente. Encuesta elaborada en el estudio Diseño de una Ruta Ecoturística en Tolú-Coveñas, 2016.

En relación con el número de personas con las que realizan los viajes, el $57 \%$ de las personas aluden que en promedio viajan con más de tres personas, el 30\% viaja con dos personas y el $11 \%$ viaja solo con una. Sin embargo, en la temporada de vacaciones, Semana Santa, los turistas denotan que viajan hasta con 10 personas ya que se reúnen tanto familias como amigos. Así mismo, con respecto a las pernoctaciones se evidencia una situación de orden similar a la cantidad de turistas que llegan a los destinos, puesto que el promedio de pernoctación fue de 4 noches.

De otro lado, el análisis precisa que se presenta afluencia de turistas en los meses de noviembre, diciembre, enero y junio con el 62\%, mientras que, en los meses de marzo, abril, mayo representa el 27\%. Panorama, que muchas veces no representa ganancias para los establecimientos de hospedaje, debido a la informalidad que se presenta en el sector y que acapara a los turistas con precios muy por 
debajo, constituyéndose como competencia desleal.

Otro aspecto importante en la demanda turística lo constituyen los intereses o motivaciones de los turistas por las actividades que ofrecen los destinos, conforme a los resultados el $64 \%$ de los turistas practican el turismo de sol y playa, mientras que el $31 \%$ practican el turismo de naturaleza.

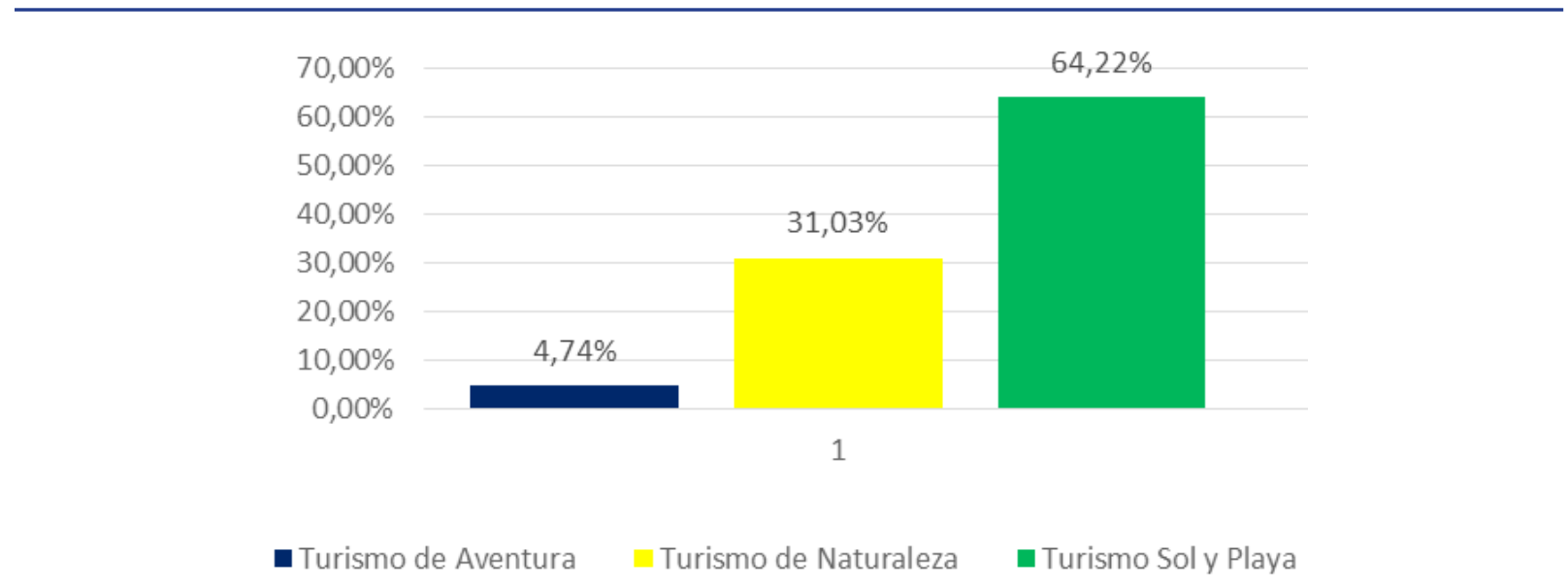

Figura 3. Principal turismo que práctica.

Fuente. Encuesta elaborada en el estudio Diseño de una Ruta Ecoturística en Tolú-Coveñas, 2016.

Situación que es consecuente con las características de los territorios, debido a la promoción de actividades enmarcadas en este turismo desde siempre. No obstante, la proporción de turistas que están dispuestos a realizar turismo de naturaleza (ecoturismo), es relevante y creciente, panorama que favorece a las zonas para ofrecer dicho turismo y aprovechar este abanico de oportunidades para el sector; teniendo como base la línea estratégica de turismo en el plan de desarrollo turístico de Sucre.

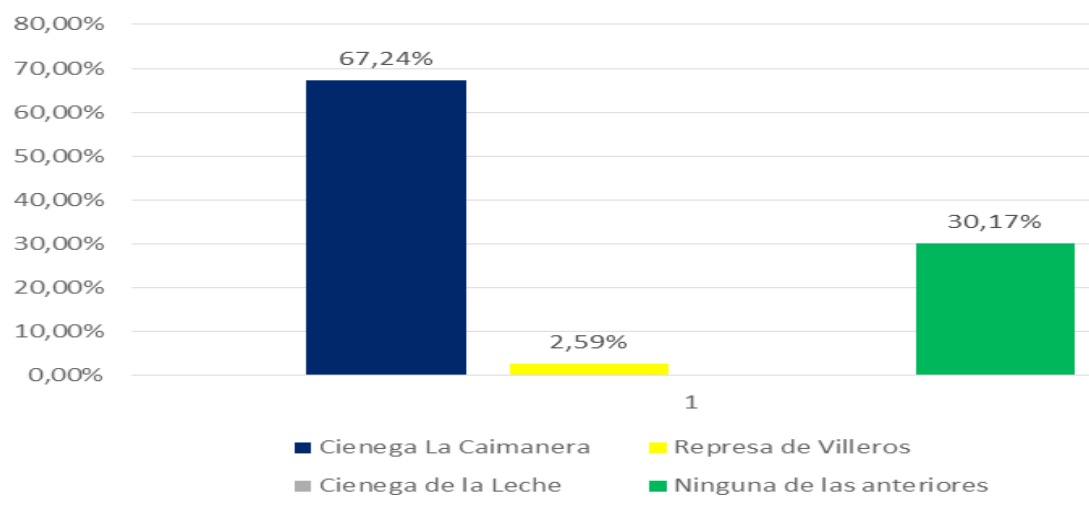

Figura 4. Áreas naturales que conocen turistas.

Fuente. Encuesta elaborada en el estudio Diseño de una Ruta Ecoturística en Tolú-Coveñas, 2016. 
Respecto al conocimiento de las áreas naturales de las zonas, la mayoría de las personas encuestadas reconoce y han visitado el área natural Ciénaga de la Caimanera con el $67,24 \%$ y el 2,59\% conoce el área natural Represa de Villeros. Mientras que el 30,17\% de los turistas no conocen ninguna de las áreas naturales del caso de estudio.

El desconocimiento de los turistas de los sitios naturales de estos territorios, es una consecuencia de la baja promoción de los recursos y/o atractivos que posee la región, a lo que se le suma la falta de visión de los municipios para brindar y desarrollar la oferta diversificada de los productos del turismo.

\begin{tabular}{|l|l|}
\hline Medios de Información & Porcentaje (\%) \\
\hline Agencia de viajes & $5,17 \%$ \\
\hline Internet & $8,62 \%$ \\
\hline Amigos & $17,24 \%$ \\
\hline Familia & $56,47 \%$ \\
\hline Redes sociales & $12,50 \%$ \\
\hline
\end{tabular}

Tabla 1. Medios de información elegidos para visitar un destino.

Fuente. Encuesta elaborada en el estudio Diseño de una Ruta Ecoturística en Tolú-Coveñas, 2016.

En cuanto a los medios de información que normalmente utilizan los viajeros, para conocer los destinos, se puede observar que el 56,47\% informa que conoce de los destinos por medio de la familia, el $17,24 \%$ mediante los amigos, seguido el $21,12 \%$ lo hace a través de las redes sociales y el internet (Tabla 1.). En menor escala consultan las agencias de viajes para solicitar este servicio con el 5,17\%. En los resultados, los turistas prefieren organizar los viajes ellos mismos $(80,17 \%)$ y solo el $19,83 \%$ aludieron que recurren a estas agencias para solicitar el servicio.

\section{Conclusiones}

La demanda de los municipios de Coveñas y Tolú, se caracteriza por estar asociada a sitios de interés particulares y a un turismo ligado a la oferta de sol y playa, frente a lo anterior se tiene que de la población estimada de 30.000 visitantes al año; el 31,03\% practican un turismo de naturaleza lo que se estima en una cifra de 9.309 turistas, que a pesar del desconocimiento de sitios turísticos y el potencial de los mismos, puede llegar a ser una gran fuente de ingreso y desarrollo para este subsector del turismo. Ahora bien, dentro de los municipios se encuentran áreas naturales importantes para la práctica de actividades del ecoturismo: Ciénaga la Caimanera, Represa de Villeros y Ciénaga de la Leche en donde se pueden realizar recorridos por los caños navegables observando la fauna y flora de las mismas, éstos dos últimos actualmente son poco conocidos por los turistas, a lo que se le suma la poca divulgación de la información que hace que de la cifra considerada de turistas que llegan a estos sitios sea menor a lo estimado y por consiguiente que la proyección futura tienda a disminuir, debido en gran parte a que los medios de comunicación y promoción de estos sitios no sean los más convencionales.

Todo lo anterior conlleva a que las áreas turísticas de los municipios de Coveñas y Tolú están subdesarrolladas y poco valoradas, en donde no se reconoce lo primordial que es la actividad en su economía y los beneficios que tendrían si actuaran de manera conjunta en espacios de colaboración y cooperación para afrontar a los competidores. Teniendo en cuenta este panorama, en el largo plazo se debe considerar los municipios como destinos ecoturísticos mediante la identidad cultural y áreas naturales características de los mismos; de igual manera se debe diversificar tanto la oferta (prestación de servicios turísticos) como la demanda turística, aprovechando las áreas con potencial y riquezas naturales, su patrimonio intangible (cultura), claro está sin dejar de lado el ámbito ambiental en la preservación y conservación para su sostenibilidad. Bajo estas circunstancias, se deben planificar los territorios como destino 
ecoturístico, promover la oferta y demanda turística eficientemente en el sector turístico con el compromiso de todos los actores de la cadena productiva del turismo. Es ahí en donde está el reto de los municipios en integrar mejoras en la planeación y gestión por la preservación y conservación de los recursos naturales, dar valor agregado a las comunidades locales, mejorar la calidad del servicio y garantizar mejores condiciones en infraestructura y servicios turisticos que complementen los destinos.

\section{REFERENCIAS}

Alburquerque, F. (1997). Metodologia para el desarollo economico local. Recuperado en: http://archivo.cepal.org/pdfs/1997/ S9720129.pdf

COMTS. (2013). Diagnostico del mercado laboral y perfil económico y productivo. Departamento de Sucre. Documento II. Recuperado en: http://www.otrabajosucre.org/pdf/Libro_Diagnostico_Mercado_Laboral_WEB.pdf

Organizacion Mundial de Turismo- OMT. (2007). Entender el turismo: glosario básico . Recuperado en: http://media.unwto. org/es/content/entender-el-turismo-glosario-basico

Rigol , L. M. \& Pérez, R. (2011). La gestión de la demanda turística como concepto. Revista en investigación en turismo y desarrollo local, 4(9). Recuperado en: http:// www.eumed.net/rev/turydes/o9/rmpc. htm

Serrano, J. C. (2011). El sector servicios en la economía global: Transformaciones y consecuencias. Clío, (37). Recuperado en: http://clio.rediris.es/n37/oposiciones2/ temao8.pdf

The International Ecotourism Society. (1990). Definición y principios del ecoturismo. Recuperado en: http://www.ecotourism. org/ties-ecotourism-espa\%C3\%B1ol

Vanegas , G. M. (2006). Ecoturismo instrumento de desarrollo sostenible. (Monografía de especializaión, Universidad de Antioquia) Recuperado en: http:// bibliotecadigital.udea.edu.co/bitstream/10495/149/1/EcoturismoInstrumentoDesarrolloSostenible.pdf 